\title{
Writing Software or Writing Scientific Articles?
}

\author{
T. Basaglia, Z. W. Bell, Member, IEEE, P. V. Dressendorfer, Fellow, IEEE, A. Larkin, and M. G. Pia
}

\begin{abstract}
An analysis of publications related to high energy physics computing in refereed journals is presented. The distribution of papers associated to various fields of computing relevant to high energy physics is critically analyzed. The relative publication rate of software papers is evaluated in comparison to other closely related physics disciplines, such as nuclear physics, radiation protection and medical physics, and to hardware publications. The results hint to the fact that, in spite of the significant effort invested in high energy physics computing and its fundamental role in the experiments, this research area is underrepresented in scientific literature; nevertheless the analysis of citations highlights the significant impact of software publications in experimental research.
\end{abstract}

\section{INTRODUCTION}

$\mathbf{P}$ UBLICATION in scholarly journals plays a fundamental role in scientific research, and has practical effects on academic careers and the evaluations performed by funding agencies.

The recent interest in open access publication [1] has motivated scientometric studies of publications in high energy physics (HEP). Quantitative data concerning physics results publications [2] in physics journals are available, but no similar analysis of high energy physics publications in technological journals has been published yet. Neither, to the authors' knowledge, has a quantitative study of software related publications in high energy physics and other radiation physics disciplines been documented in literature.

This paper presents a quantitative analysis of publications relating to computing in high energy physics. It compares publication rates for hardware and software related papers, evaluates distributions and trends for several different computing and software domains, and looks at the impact of software papers as detemined by citation statistics.

\section{DAta SOURCES AND ANALYSIS MethoD}

The main source of data for this study is the ISI Web of Science [3]. It covers the period since 1990 to the presentt date and provides a set of tools for searching the database and analyzing the search results. Other publication databases,

Manuscript received November 23, 2007.

T. Basaglia is with CERN, CH-1211 Geneva 23, Switzerland.

Z. W. Bell is with the Oak Ridge National Laboratory, Oak Ridge TN 37830; Oak Ridge National Laboratory is managed by UT-Battelle, LLC, for the U.S. Department of Energy under Contract DE-AC05-00OR22725.

P. V. Dressendorfer is with IEEE Transactions on Nuclear Science (TNS) (e-mail: p.dressendorfe@ieee.org).

A. Larkin is with the IEEE, Piscataway, NJ 08854-4141 USA.

M. G. Pia is with INFN Sezione di Genova, 16146 Genova, Italy (e-mail: MariaGrazia.Pia@ge.infn.it).

Color versions of one or more of the figures in this paper are available online at http://ieeexplore.ieee.org.

Digital Object Identifier 10.1109/TNS.2008.919563 like Google Scholar and INSPEC [4], were utilized to retrieve complementary information, for instance articles published prior to 1990, and for cross-checking the analyses based on the ISI Web of Science.

A general analysis of computing-related papers in particle physics and similar research fields has to cope with the practical difficulty of identifying relevant papers in the publication databases: keyword searches based on generic criteria, like "computing" or "software" are prone to introduce a large number of non-pertinent papers in the selection, while the large size of data samples thus generated makes them unmanageable for detailed analysis. Therefore this study confined itself to a few well defined areas, representing significant domains in the field: the comparison of hardware and software oriented publications in technological journals produced by high energy physics experiments, the analysis of relevant software domains such as grid computing and simulation, and the evaluation of the impact of software publications in experimental life through an analysis of paper citations in scholarly journals.

Data samples were selected by means of keyword searches, exploiting search configuration options available through the database user interface. Candidate keywords were first tested on controlled samples extracted from the IEEE TRANSACTIONS ON NUCLEAR SCIENCE (TNS) publication database, to which the authors have direct access. This procedure allowed an evaluation of the suitability of keywords for larger scale analysis, according to the fraction of publications correctly identified and the amount of non-relevant ones introduced in the selected sample.

The search criteria for the identification of data samples were refined in the course of the analysis based on the comparison of results from different databases: this iterative method helped optimize the completeness and reliability of the data subject to further analysis.

A few analyses were performed by comparing the results obtained from the ISI Web of Science and INSPEC. It has to be stressed that INSPEC covers physics, electronics and computing, whereas The ISI Web of Science covers all scientific domains; the differences in subject indexing between the two databases are considerable. This helped to identify appropriate search strategies and to evaluate possible systematic errors or biases introduced in the results; the databases provided consistent results.

The data sets resulting from automated searches were subjected to manual inspection to evaluate the amount of noise (i.e. number of non-relevant papers) introduced in the sample and to further classify the collected publications according to more detailed criteria. In some cases, like the technological literature of high energy physics experiments in Section III and the citation statistics reported in Section VII-C, the whole data set was manually inspected; in other cases only a subset of the data was 


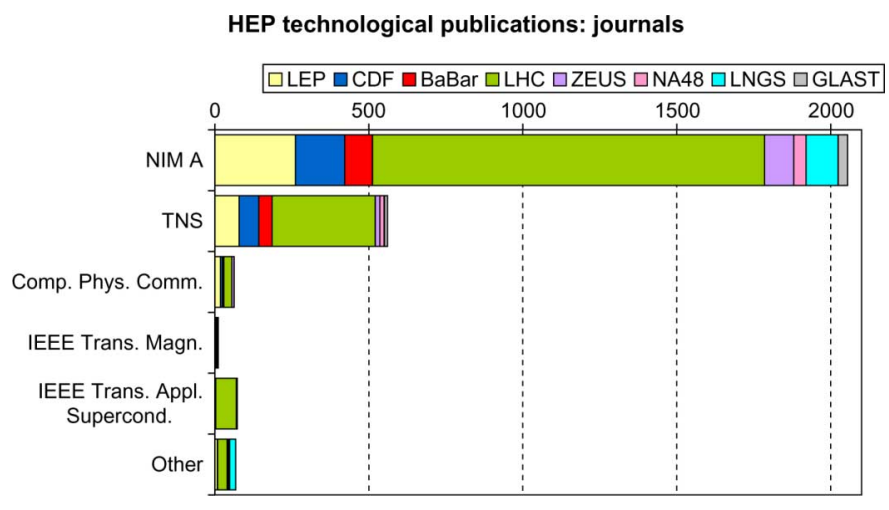

Fig. 1. Journals where the technological papers of representative high energy physics experiments are published.

evaluated manually and the reliability of the sample was extrapolated to the whole data set. We do not think that the amount of noise and the incompleteness of the data samples deriving from automated searches affect the conclusions of the various analyses; the uncertainties of the results as determined from manual inspection are smaller than $5 \%$.

Most of the analyses reported concern papers published between 2002 and 2006 as a representative period of recent research activity in the domain considered; the extension (five years) is sufficiently wide to avoid significant bias in the results due to fluctuations in the publication rates or characteristics. The citation analysis concerns the whole ISI Web of Science coverage since 1990. Whenever other time constraints were applied, they are specifically indicated in the following sections.

\section{HEP EXPERIMENTS: HARDWARE AND SOFTWARE PUBLICATIONS}

An analysis was performed to evaluate the distribution of technological publications in high energy physics experiments over hardware and software related topics. The period covered by the analysis extends up to 2006 .

The study was performed on a set of experiments representative of high energy physics research in the past two decades: the four LEP experiments (ALEPH [5], DELPHI [6], L3 [7] and OPAL [8]), the fixed target NA48 [9] experiment and the LHC experiments (ALICE [10], ATLAS [11], CMS [12] and LHCb [13]) at CERN, CDF [14] at FNAL, ZEUS [15] at DESY and BaBar [16] at SLAC. Astroparticle experiments were represented in the sample by GLAST [17] and by experiments at the Gran Sasso Laboratory (LNGS).

The experiments' publications in only technological journals were considered; as it can be observed in Fig. 1, the majority of such papers appears to be clustered in two journals, Nuclear Instruments and Methods (NIM) A and IEEE TRANSACTIONS ON NUCLEAR SCIENCE. These publications were classified in three categories: "Hardware", "Software" and "Trigger-Data Acquisition". The third category reflects the evolution of this domain: it used to be more hardware-oriented in the earlier high energy physics experiments considered, while it is more software-oriented in the current-generation ones. The attribution of papers to each category was based on the detailed inspection of the title and abstract of the paper, or the whole text in case of ambiguities; therefore it was subject to a certain degree of subjectivity. In the case of uncertainty between hardware and software classification, the latter was always chosen, resulting in a uniform bias of the results.

The distribution of publications across the three categories is shown in Fig. 2, and the ratio between hardware and software papers is highlighted in Fig. 3. The results show that far fewer software (as compared to hardware) publications are consistently produced by high energy physics experiments; Fig. 3 suggests a trend in which the publication of hardware versus software papers even seems to increase from the earlier generation of LEP experiments to the current generation of LHC ones.

\section{GRID COMPUTING}

Grid computing is in an emerging technology in the field of distributed computing. In the past few years it has evolved from a conceptual prototyping stage to an operative one, with several computational grid systems now actively in production mode. Grid computing is essential to the next generation high energy physics experiments at LHC. Due to the amount of data produced and the complexity of their elaboration, the conventional computing schemes adopted in previous generations' experiments would not be adequate to the scale of the new experiments, and worldwide distributed computing has become a necessity to cope with the unprecedented scale of experimental demands. The crucial role played by grid computing in high energy physics experiments has motivated large investments of funds and manpower into this domain in the recent years.

Grid computing is a multidisciplinary domain. It encompasses the development of grid infrastructure as well as its application in various scientific domains.

A few specialized journals are dedicated to grid computing, although papers related to this field are also published in journals characterized by a broader coverage of distributed computing research. The field is very active in terms of number of publications: 4572 papers in total have been published in specialized grid and distributed computing journals over the 2002-2006 period.

Given the intrinsically distributed nature of grid computing and the ongoing international effort for the development of large scale grids, the geographical distribution of published papers in this field is of interest. Fig. 4 shows the number of papers published in distributed computing and specialized grid journals during the years 2002-2006. The statistics derive from a search in the ISI Web of Science articulated around the "grid computing" keyword and multiple variants of it; it covers 23 refereed journals in the field as well as conference proceedings indexed in the same database, related to conferences or workshops specific to this field. A paper is considered associated to a geographical region whenever at least one of its co-authors is affiliated to an institute located in that region.

The geographical distribution of publications exhibits significant differences between refereed journals and conference proceedings. Fig. 5 and Fig. 6 report the statistics of papers by institute over the years 2002-2006 in the two cases; they list the institutes ranked in the first 10 positions based on 
HEP experiments: technological publications

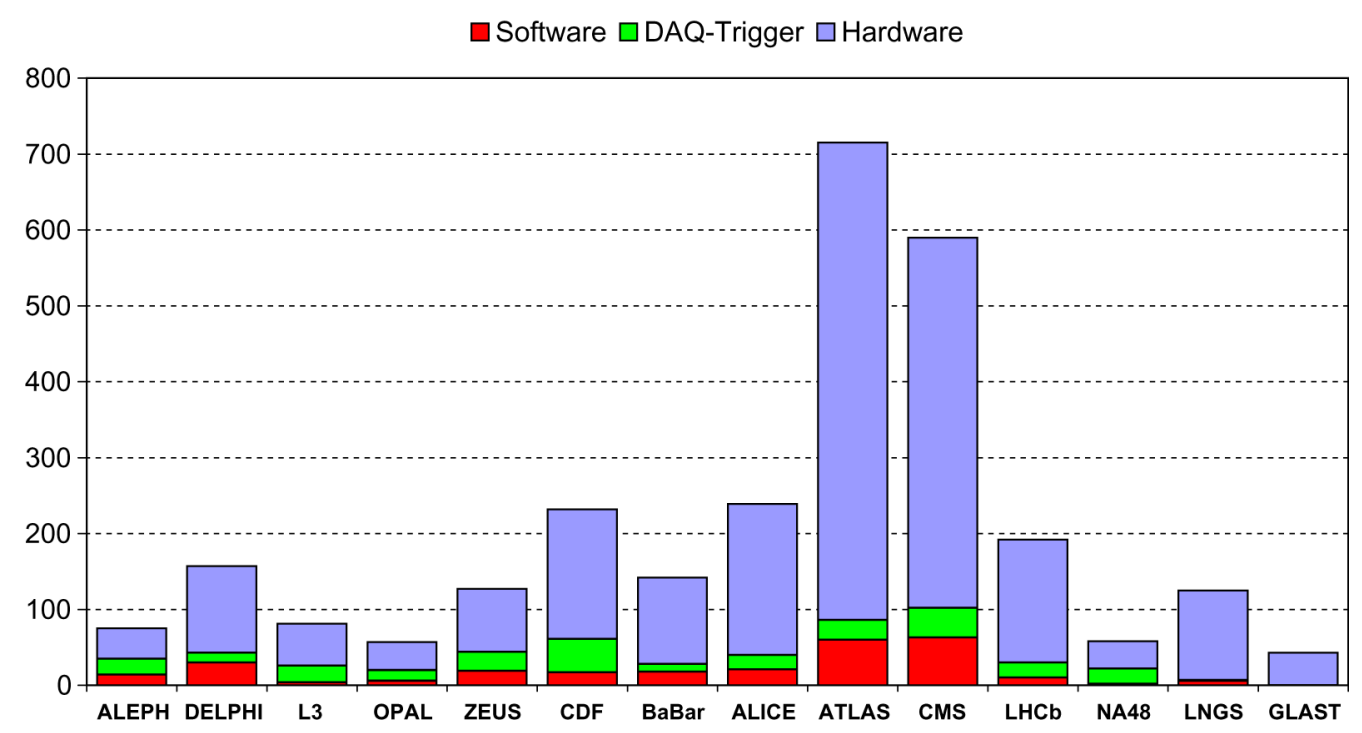

Fig. 2. Publications in technological journals of representative high energy physics experiments; the number of hardware, software and trigger-data acquisition papers are plotted for each experiment.

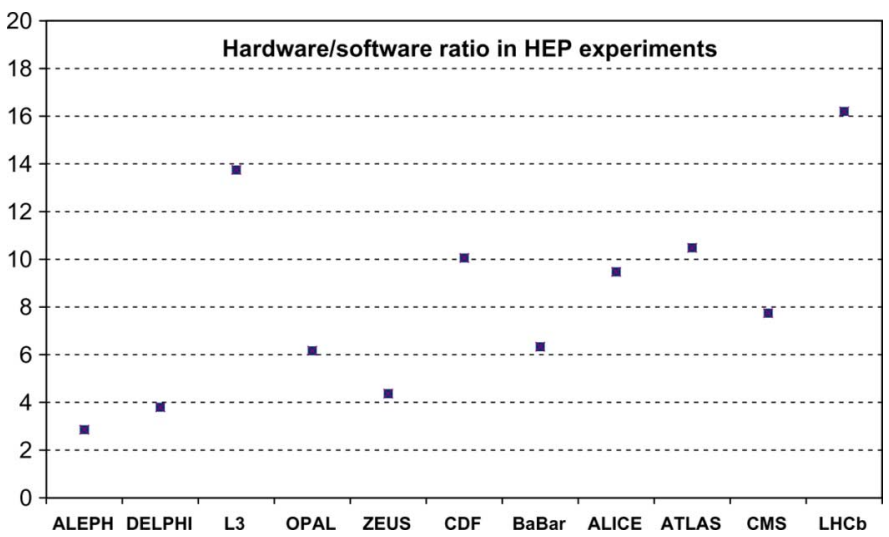

Fig. 3. The ratio of hardware over software publications in technological journals of representative high energy physics experiments.

Grid computing: geographical distribution of papers

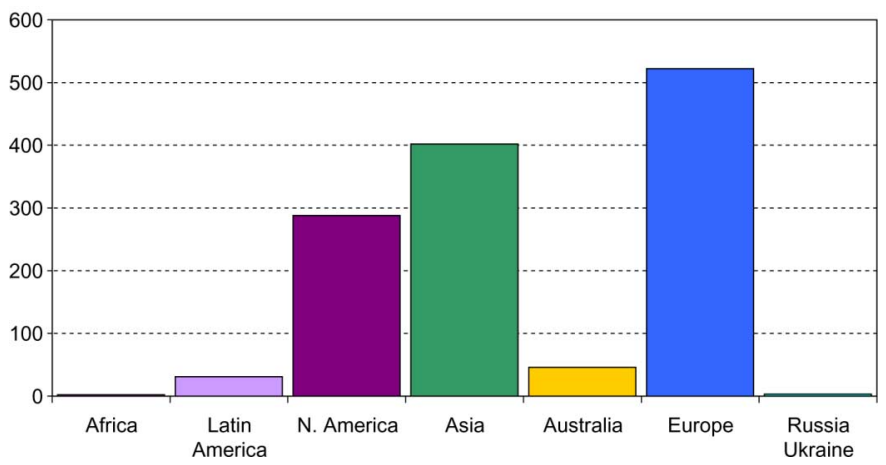

Fig. 4. Geographical distribution of grid computing papers; the plot reports the number of papers published in the years 2002-2006 associated to each region considered.

the number of publications. There is a striking difference in the publication patterns, with Asian universities dominating publications in conference proceedings and North American
Grid - Top 10 institutes, Computing journals

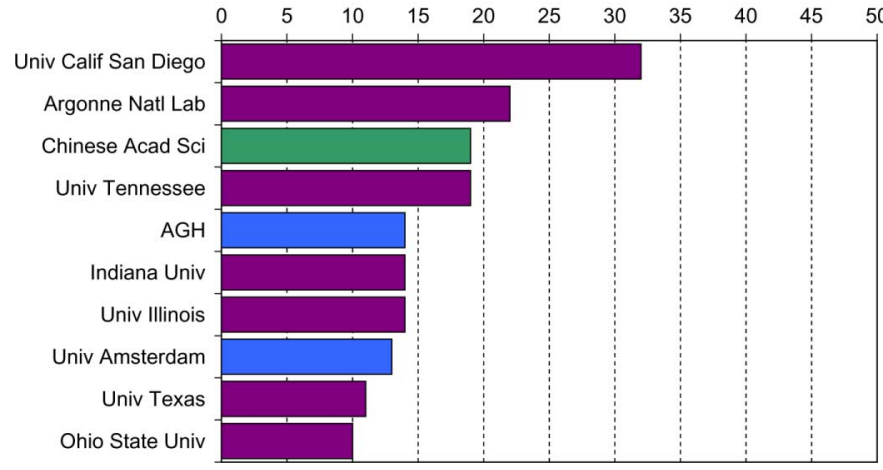

Fig. 5. Grid computing publications in refereed journals specific to distributed computing: number of papers published in the years 2002-2006 ranked by institute affiliation; the plot reports the institutes ranked in the first 10 positions.

institutions dominating those in refereed journals. This hints at a different emphasis in the two regions for the preferred publication path.

Fig. 7 shows the same distribution as in Fig. 5 extended to include publications in IEEE TRANSACTIONS ON NUCLEAR SCIENCE, which was identified in Section III as one of the preferred target journals for high energy physics technological publications. In this context high energy physics laboratories, such as CERN and FNAL, and a national institute active in high energy physics research (INFN) appear to play a significant role. The difference with respect to the distribution in Fig. 5 suggests that high energy physicists may prefer to publish grid-related research results in journals well known to their scientific community rather than in specialized computing journals. It is worth noting that all the publications concerning grid computing in Nuclear Instruments and Methods A over the same period in issues devoted solely to conference proceedings. 
Grid - Top 10 institutes, Proceedings

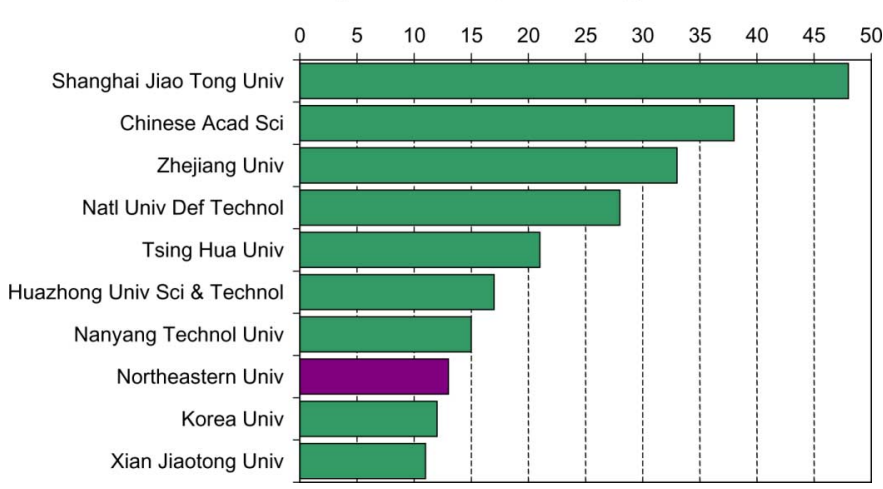

Fig. 6. Grid computing publications in conference or workshop proceedings specific to distributed computing: number of papers published in the years 2002-2006 ranked by institute affiliation; the plot reports the institutes ranked in the first 10 positions.

Grid - Top 10 institutes, Computing journals + IEEE TNS

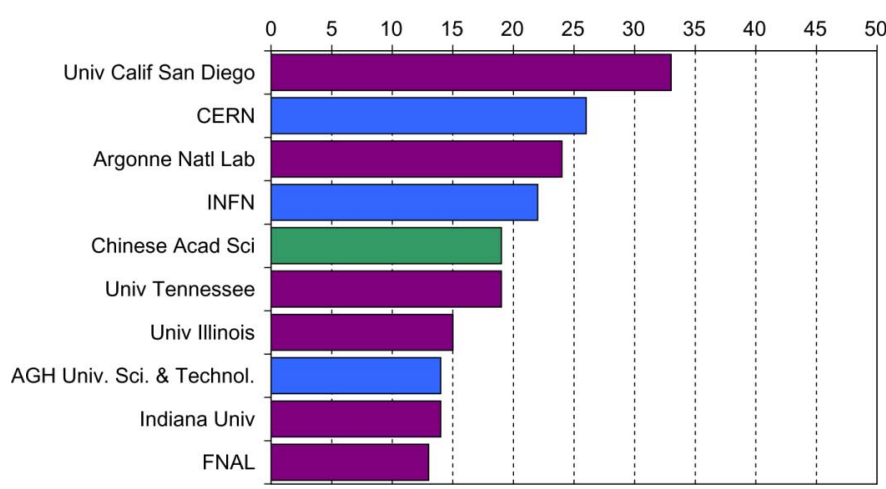

Fig. 7. Grid computing publications in refereed journals specific to grid computing, plus the IEEE TRANSACTIONS ON NUCLEAR SCIENCE: number of papers published in the years 2002-2006 ranked by institute affiliation; the plot reports the institutes ranked in the first 10 positions.

\section{Simulation}

Simulation plays a fundamental role in various aspects of the lifecycle of high energy physics experiments: detector design, physics reach evaluation, development and optimization of data reconstruction and analysis software, physics analysis, etc. It also contributes significantly to the experimental design and physics understanding in other disciplines, such as nuclear physics, medical physics and radiation protection.

Fig. 8 shows the distributions of publications mentioning or citing major Monte Carlo systems: EGS [18], [19] FLUKA [20], [21], GEANT [22], Geant4 [23], [24] MCNP [25]-[28] and Penelope [29]. These codes are representative of widely used Monte Carlo systems in various application disciplines. The data sample covers the period 2002-2006; the statistics were built on the basis of citations to reference publications in refereed journals, whenever available, or by keyword searches in the ISI Web of Science database. It should be noted that in the case of FLUKA and Penelope a significant fraction $(35 \%$ and $26 \%$ respectively) of the entries in the plot is represented by self-citations, i.e. publications by developers or maintainers of

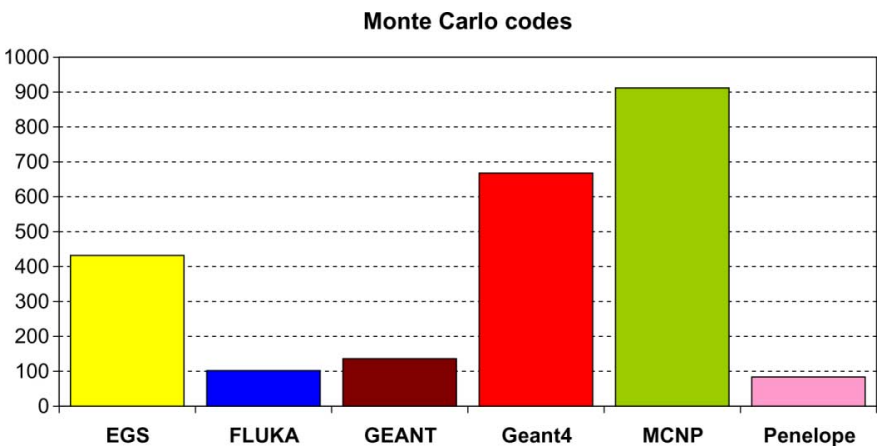

Fig. 8. Number of publications mentioning or citing selected major Monte Carlo codes in the period 2002-2006.

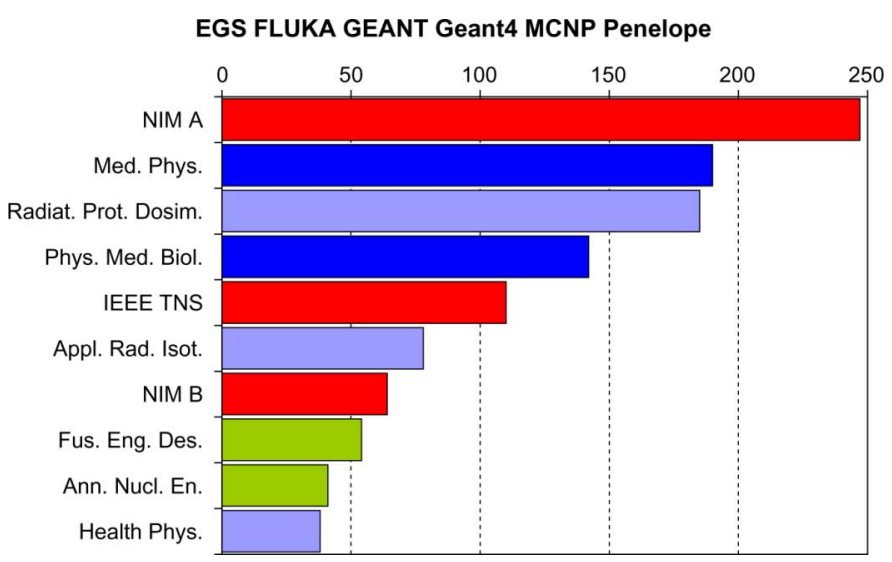

Fig. 9. Number of articles mentioning or citing selected major Monte Carlo codes sorted by journal; the statistics refers to years 2002-2006.

these Monte Carlo codes, while self-citation is negligible for the more widely used systems.

The journals where the majority of these papers are published are shown in Fig. 9: they include multi-disciplinary journals, like Nuclear Instruments and Methods and IEEE TRANSACTIONS ON NUCLEAR SCIENCE, as well as specialized ones, like Medical Physics or Radiation Protection Dosimetry, devoted to specific disciplines.

The distribution of papers in the same set of journals resulting from the generic keyword search "Monte Carlo or simulation" is shown in Fig. 10; publications in multi-disciplinary technological journals were classified according to their pertinent field of application whenever it could be easily ascertained unambiguously. High energy physics papers represent a fraction of the simulation papers published in Nuclear Instruments and Methods and IEEE TRANSACTIONS ON NUCLEAR SCIENCE, while the vast majority of simulation publications is associated with other disciplines, like medical physics, nuclear physics and radiation protection.

HEP experiments appear to publish a relatively small number of papers related to Monte Carlo simulation, despite its crucial role in the experiment and the major effort usually invested by such experiments to develop detailed simulations of their complex detectors. A case study performed on CDF vertex detector resulted in 79 papers concerning the detector hardware, 11 papers concerning its associated trigger and data acquisition 


\section{Monte Carlo / Simulation}

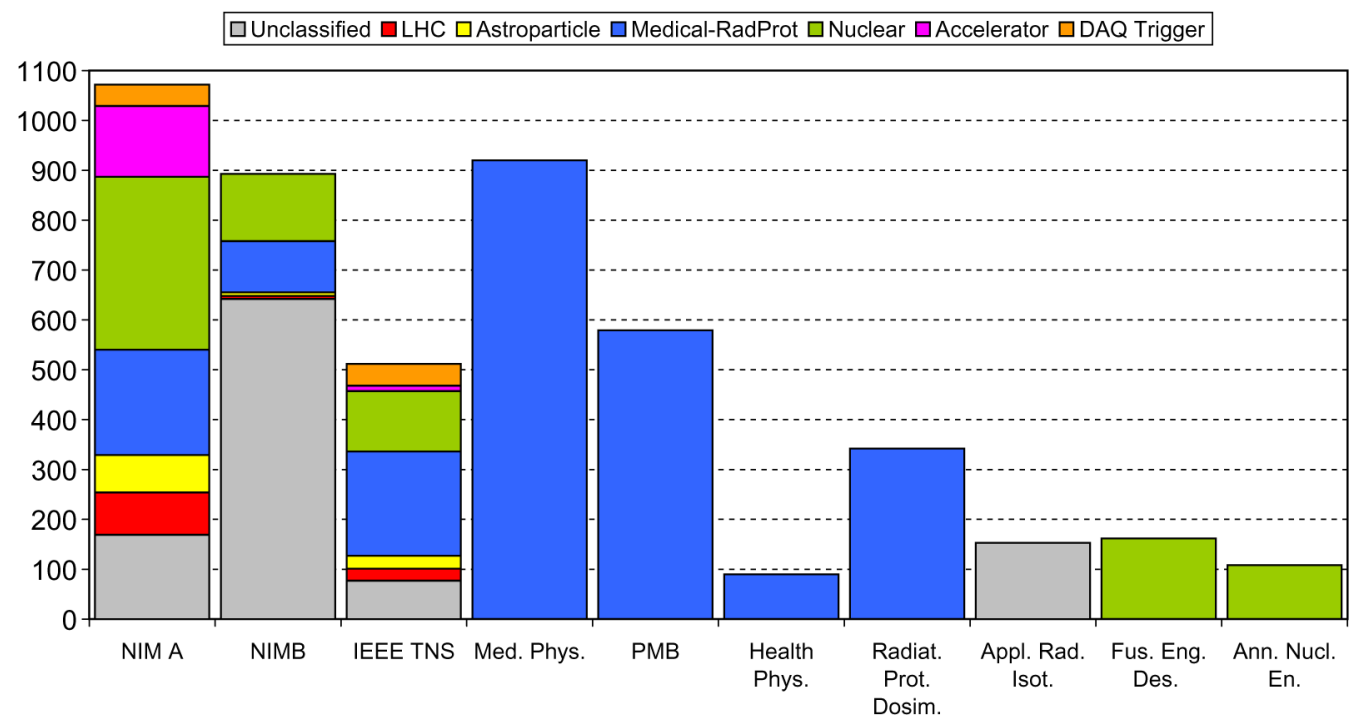

Fig. 10. Distribution of simulation articles within different journals; the statistics refers to years 2002-2006.

system, one paper on the vertex reconstruction software and no papers on the detector simulation. LEP experiments published a few simulation papers in total; however, it is worth stressing that the only paper published by a LEP experiment as a comprehensive documentation of its simulation software [30] collected 324 citations, thus demonstrating the significant role played by simulation in the experiment. The other LEP experiments developed simulation systems of comparable complexity and capabilities; nevertheless, they do not appear to have been published in refereed journals. Other disciplines appear more active in publishing papers related to simulation: for instance, approximately 1500 articles related to this topic were published in Medical Physics and Physics in Medicine and Biology (PMB) in the period 2002-2005.

\section{TRENDS IN SOFTWARE PUBLICATIONS}

As previously mentioned, a generic search for software or computing related papers in a large scale publication database is prone to produce unreliable and unmanageable results.

A generic search for computing related papers was performed on a subset of technological journals to evaluate the evolution of the fraction of such publication as a function of time. The generic search included "software", "computing" or "algorithm" as keywords, and was performed over the ten journals with the highest impact factor in the Nuclear Science and Technology category. A comparative evaluation of the results over the entire period covered by the ISI Web of Science and the years 2002-2006, documented in Fig. 11, shows that the fraction of computing related publications has increased in the recent years in several journals.

\section{Citation Statistics}

A metric to evaluate the impact of software publications is represented by the number of citations they receive. The absolute number of citations collected, as well as the relative position

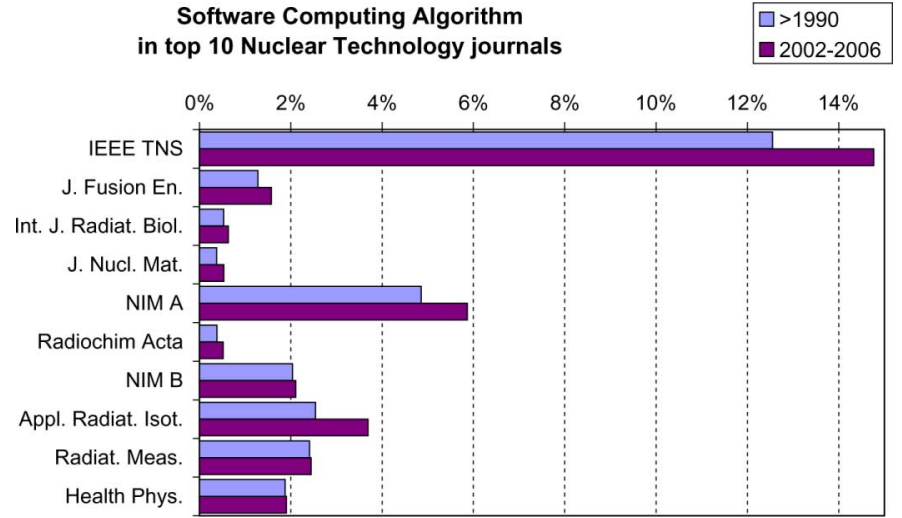

Fig. 11. Fraction of computing papers in representative technological journals in the period 1990-2006 and 2002-2006: the publication of computing papers has increased in most journals in the recent years.

with respect to other papers, are relevant parameters. The citation statistics reported in this paper were derived from the ISI Web of Science and concern the period covered by this tool up to 25 October 2007.

\section{A. High Energy Physics}

The citations collected by publications produced by major laboratories and institutes in this field (i.e. involving authors affiliated to them) constitute a parameter to identify their scientific impact in the associated research community. CERN and INFN were considered in a case study, as representative of an international laboratory and a national institute.

The five most cited CERN and INFN papers are listed in Tables I and II respectively (excluding the periodic reviews of particle properties); in both cases two software papers appear among them. These results highlight the relevant role played by and impact of software in high energy physics, in spite of the 
TABLE I

The Five Most CITED CERN Publications

\begin{tabular}{|c|c|c|c|}
\hline Title & Year & Reference & Citations \\
\hline High-energy-physics event generation with Pythia-5.7 and Jetset-7.4 & 1994 & [31] & 1835 \\
\hline A possible new dimension at a few $\mathrm{TeV}$ & 1990 & [32] & 981 \\
\hline Comparison of grand unified theories with electroweak and strong coupling-constants measured at LEP & 1991 & [33] & 801 \\
\hline GEANT4 - a simulation toolkit & 2003 & [23] & 663 \\
\hline Radiative corrections to the masses of supersymmetric Higgs bosons & 1991 & [34] & 630 \\
\hline
\end{tabular}

TABLE II

The Five Most Cited INFN Publications

\begin{tabular}{|c|c|c|c|}
\hline Title & Year & Reference & Citations \\
\hline Stochastic resonance & 1998 & {$[35]$} & 1574 \\
\hline HERWIG 5.1 - A Monte-Carlo event generator for simulating hadron emission reactions with interfering gluons & 1992 & [36] & 999 \\
\hline Observation of top-quark production in $\bar{p} p$ collisions with the Collider Detector at Fermilab & 1995 & [37] & 739 \\
\hline GEANT4 - a simulation toolkit & 2003 & [23] & 663 \\
\hline Radiative corrections to the masses of supersymmetric Higgs bosons & 1991 & [34] & 630 \\
\hline
\end{tabular}

TABLE III

The Five Most Cited Publications in NuCl. Instrum. Meth. A

\begin{tabular}{|c|c|c|c|}
\hline Title & Year & Reference & Citations \\
\hline GEANT4 - a simulation toolkit & 2003 & {$[23]$} & 663 \\
\hline ESCL8R and LEVIT8R - Software for interactive graphical analysis of HPGe coincidence data sets & 1995 & [41] & 491 \\
\hline The CLEO-II detector & 1992 & [42] & 453 \\
\hline The construction of the L3 experiment & 1990 & [7] & 450 \\
\hline The OPAL detector at LEP & 1991 & [8] & 442 \\
\hline
\end{tabular}

TABLE IV

The Five Most Cited Publications In The Nuclear Science and Technology Category

\begin{tabular}{|c|c|c|c|}
\hline Title & Year & Reference & Citations \\
\hline GEANT4 - a simulation toolkit & 2003 & [23] & 663 \\
\hline Guidelines for limiting exposure to time-varying electric, magnetic, and electromagnetic fields (up to $300 \mathrm{GHz}$ ) & 1998 & [43] & 547 \\
\hline Luminescence dating of quartz using an improved single-aliquot regenerative-dose protocol & 2000 & [44] & 499 \\
\hline ESCL8R and LEVIT8R - Software for interactive graphical analysis of HPGe coincidence data sets & 1995 & [41] & 491 \\
\hline The CLEO-II detector & 1992 & [42] & 453 \\
\hline
\end{tabular}

relatively small number of software papers among the technological publications of high energy physics experiments.

\section{B. Technological Journals}

The citation analysis was articulated by two approaches: technological journals representative of specific fields were considered, as well as the citation statistics of the whole Nuclear Science and Technology category defined by the Journal Citation Reports [38]. The specific analyses concerned the two journals most representative of high energy physics technological publications (Nuclear Instruments and Methods A and IEEE TRANSACTIONS ON NUCLEAR SCIENCE), two highly representative journals in medical physics (Medical Physics and Physics in Medicine and Biology) and radiation protection journals.

The five most cited publications in Nuclear Instruments and Methods A, the technological journal identified as the most popular in high energy physics, are reported in Table III; two software papers appear on top of the list. They are followed by three articles describing large scale high energy physics detectors: in these cases a large number of references (for instance, $88 \%$ for the CLEO-II detector paper) derives from other publications by the same experiment. The most cited paper of this journal concerning a specific hardware topic [39] is ranked in seventh position with 367 citations.
No papers on software topics appear in the equivalent list of the five most cited publications of IEEE TRANSACTIONS ON NUCLEAR SCIENCE.

A software paper [40] is in second position among the most cited publications in the medical physics journals Medical Physics and Physics in Medicine and Biology: it collected 391 citations, while the most cited one in this domain was referenced 610 times. This outcome confirms the significant role played by Monte Carlo simulation in medical physics, consistent with the results documented in Section V. The citation statistics of radiation protection journals do not include papers on software topics among the top five publications.

The most cited publication in the whole Nuclear Science and Technology category is a software paper, and another software article is ranked in fourth position; the results of the citation analysis are listed in Table IV. The category encompasses 32 journals and more than 132000 papers published since 1990.

\section{A Case Study: Geant4 Citations}

A detailed analysis of the citation of software papers provides information about the usage of the associated software tools in experimental applications. As an example, it was performed in relation to the Geant 4 reference article [23]: due to the large number of citations received, it can be considered a representative sample of widely used scientific software. 


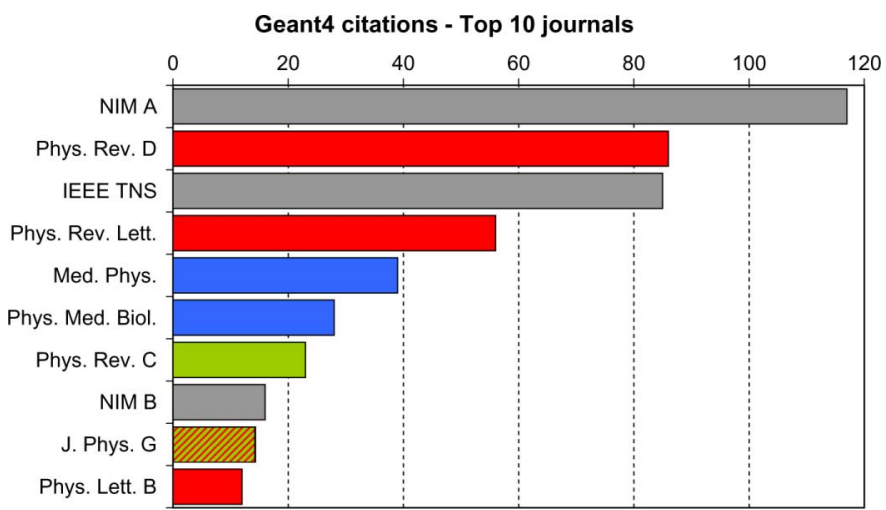

Fig. 12. Number of articles citing the Geant 4 reference paper [23] sorted by journal.

Fig. 12 shows the distribution of citations among the ten most frequently citing journals, that encompass $72 \%$ of the total number of citations. Both multi-disciplinary journals, like Nuclear Instruments and Methods and IEEE TRANSACTIONS ON NUCLEAR SCIENCE, and specialized journals in medical physics, radiation protection and nuclear physics collect a large number of Geant 4 citations. Geant 4 development was originally motivated by high energy physics requirements and a large number of its developers are affiliated with high energy physics laboratories and institutes; the citation results show that Geant4 user community extends far beyond high energy physics.

Proper reference to previous research developments and results is considered an essential practice in scholarly publications. The citation patterns of papers mentioning Geant 4 in Nuclear Instruments and Methods A and IEEE TRANSACTIONS ON NUCLEAR SCIENCE have been analyzed; these two journals have been selected as the ones publishing the largest number of computing papers in the Nuclear Science and Technology category; morever they both published Geant 4 reference papers [23] and [24].

The occurrence of the correct Geant4 bibliographical [23] was verified in papers mentioning Geant 4 published since 2005: this conservative time limit ensures that the papers went through the peer review process and were published after the publication of the reference itself. Similarly, the citation of [24] was verified in papers published at least one year later. It is worth reminding the reader that the two Geant 4 references are recalled in the home page of Geant 4 web site; therefore, any user downloading the code ought to be aware of them.

Fig. 13 shows the number of papers correctly citing Geant 4 references, omitting any citation despite mentioning Geant 4 , and including incorrect or incomplete citations. It is evident that Geant 4 is not properly cited in many cases.

A similar analysis was performed on publications in Nuclear Instruments and Methods A mentioning GEM detectors: only $8 \%$ of them did not include proper references. These results hint to a different perception in the experimental community of hardware and software publications as scientific research references.

\section{CONCLUSION}

This study documents the first quantitative analysis of software publications in high energy physics and other radiation physics domains.
Geant4 references 2005-2006

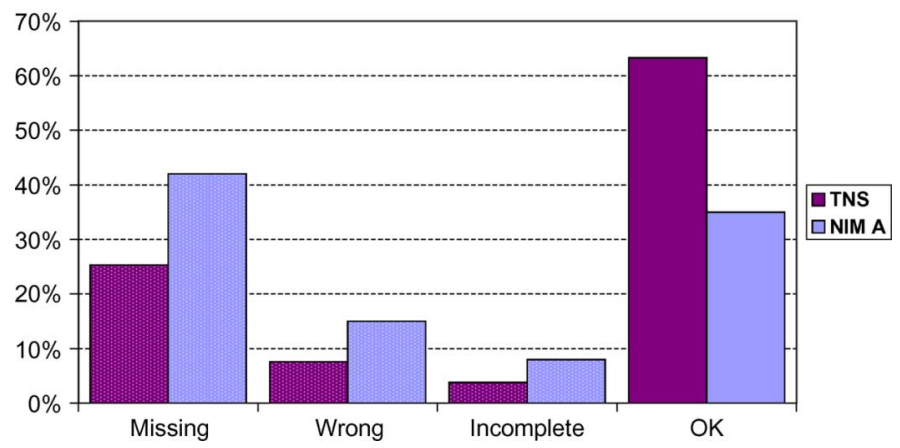

Fig. 13. Citation pattern concerning the Geant 4 reference article in Nuclear Intruments and Methods A and IEEE TRANSACTIONS ON NUCLEAR SCIENCE: number of papers with correct, incomplete, wrong or omitted references. [23]

The results show an overwhelming number of hardware papers published by high energy physics experiments with respect to software ones; nevertheless, the large number of citations received by software papers in this field demonstrates the key role of software research and development in particle physics experiments.

The quantitative evaluation of computing publications highlights peculiar features of software related publications: the noticeable difference in the geographical distribution of grid computing publications in refereed journals and conference proceedings, and the apparent low number of simulation publications in high energy physics compared to other disciplines, despite the significant role played by simulation in high energy physics experiments.

The analysis of citations produced the surprising result that a software paper originating from high energy physics [23] is the most cited publication in the whole Nuclear Science and Technology category.

The results of this study suggest that software is underrepresented in high energy physics literature in spite of its significant contribution to the advancement of the field demonstrated by the large number of citations received by software papers. HEP computing seems also to be largely absent from the current debate on Open Access publishing in high energy physics. The picture emerging from these considerations induces a perception of computing in high energy physics as a support service rather than a scientific research domain.

Due to its relevance in experimental research, a wider presence of particle physics software in technological literature would be desirable. By providing a quantitative awareness of the status in the field, hopefully the present study will contribute to promoting more active publication rates in the software-oriented experimental physics community.

\section{ACKNOWLEDGMENT}

The authors are grateful to Alexander Howard, Jürgen Knobloch, Salvatore Mele, Jens Vigen and Joanne Yeomans for valuable comments and suggestions.

\section{REFERENCES}

[1] P. O. Brown et al., Bethesda Statement on Open Access Publishing, 2003 [Online]. Available: http://www.earlham.edu/ peters/fos/bethesda.htm 
[2] S. Mele, D. Dallman, J. Vigen, and J. Yeomans, "Quantitative analysis of the publishing landscape in high-energy physics," J. High Energy Phys., vol. 12, p. S01, 2006.

[3] The Thomson Corporation, ISI Web of Knowledge [Online]. Available: http://scientific.thomson.com/products/wos

[4] [Online]. Available: The Institution of Engineering and TechnologyINSPEC [Online]. Available: http://www.theiet.org/publishing/inspec

[5] W. Wu, "The ALEPH experiment at LEP," Int. J. Mod. Phys. A, vol. 1, no. 1 , pp. 117-133, 1986

[6] P. Aarnio et al., "The DELPHI detector at LEP," Nucl. Instrum. Methods Phys. Res. A, vol. A303, no. 2, pp. 209-395, 1991.

[7] B. Adeva et al., "The construction of the L3 experiment," Nucl. Instrum. Methods Phys. Res. A, vol. A289, no. 1-2, pp. 35-102, 1990.

[8] K. Ahmet et al., "The OPAL detector at LEP," Nucl. Instrum. Methods Phys. Res. A, vol. A305, no. 2, pp. 275-319, 1991.

[9] G. Anzivino, "The NA48 experiment at the CERN SPS," Nucl. Phys. B. Proc. Suppl., vol. 78, no. 1, pp. 58-63, 1999.

[10] C. Kuhn et al., "The ALICE experiment at LHC," Nucl. Phys. A, vol. 787, no. 1-4, pp. 19-28, 2007.

[11] I. Efthymiopoulos, "Overview of the ATLAS detector at LHC," Acta Phys. Pol. B, vol. 30, no. 11, pp. 2309-2329, 1999.

[12] K. Ahmet et al., "CMS, a general purpose detector for the LHC," Nucl. Instrum. Methods Phys. Res. A, vol. A344, no. 1, pp. 1-10, 1994.

[13] O. Schneider et al., "Overview of the LHCb experiment," Nucl. Instrum. Methods Phys. Res. A, vol. A446, no. 1, pp. 213-221, 2000

[14] F. Abe et al., "The CDF detector: an overview," Nucl. Instrum. Methods Phys. Res. A, vol. A271, no. 3, pp. 387-403, 1988.

[15] U. Holm et al., "The ZEUS detector," Status Rep. DESY. Hamburg, Germany [Online]. Available: http://www-zeus.desy.de/bluebook/bluebook.html

[16] B. Aubert et al., "The BaBar detector," Nucl. Instrum. Methods Phys. Res. A, vol. A479, no. 1, pp. 1-116, 2002.

[17] P. F. Michelson, "GLAST: A detector for high-energy gamma rays," in Proc. SPIE, Denver, CO, 1996, vol. 2806, pp. 31-40.

[18] H. Hirayama, Y. Namito, A. F. Bielajew, S. J. Wilderman, and W. R. Nelson, "The EGS5 code system," Stanford Linear Accelerator Center, Stanford, CA, Rep. SLAC-R-730, 2006.

[19] I. Kawrakow and D. W. O. Rogers, "The EGSnrc code system: Monte Carlo simulation of electron and photon transport," NRCC Rep. PIRS701, Sep. 2006.

[20] A. Ferrari, P. R. Sala, A. Fassò, and J. Ranft, "Fluka: A multi-particle transport code," Geneva, Switzerland, Rep. CERN-2005-010, INFN/TC-05/11, SLAC-R-773, Oct. 2005.

[21] A. Fassò et al., "The physics models of FLUKA: Status and recent developments," presented at the Computing in High Energy and Nuclear Physics 2003 Conf. (CHEP2003), La Jolla, CA, Mar. 2003, MOMT005.

[22] GEANT CERN Program Library, Long Writeup W5013, Mar. 1994.

[23] S. Agostinelli et al., "GEANT4-A simulation toolkit," Nucl. Instrum. Methods Phys. Res. A, vol. A506, no. 3, pp. 250-303, 2003.

[24] J. Allison et al., "Geant4 Developments and Applications," IEEE Trans. Nucl. Sci., vol. 53, no. 1, pp. 270-278, Feb. 2006.

[25] X-5 Monte Carlo Team, MCNP-A General Monte Carlo N-Particle Transport Code, Version 5, Los Alamos National Laboratory, Rep. LA-UR-03-1987, Apr. 2003, Rev. Mar. 2005.
[26] R. A. Forster et al., "MCNP Version 5," Nucl. Instrum. Methods Phys. Res. B, vol. B213, pp. 82-86, 2004.

[27] J. S. Hendricks et al., MCNPX 2.3.0 User's Guide (Unrestricted Distribution Version) Los Alamos National Laboratory, Rep. LA-UR-022607, Apr. 2002

[28] J. S. Hendricks et al., MCNPX, Version 26c Los Alamos National Laboratory, Rep. LA-UR-06-7991, Dec. 2006.

[29] J. Baro, J. Sempau, J. M. Fernández-Varea, and F. Salvat, "PENELOPE, an algorithm for Monte Carlo simulation of the penetration and energy loss of electrons and positrons in matter," Nucl. Instrum. Methods Phys. Res. B, vol. B100, no. 1, pp. 31-46, 1995.

[30] J. Allison et al., "The detector simulation program for the OPAL experiment at LEP," Nucl. Instrum. Methods Phys. Res. A, vol. A317, no. 1-2, pp. 47-74, 1992.

[31] T. Sjostrand, "High-energy-physics event generation with Pythia-5.7 and Jetset-7.4," Comp. Phys. Comm., vol. 82, no. 1, pp. 74-89, 1994.

[32] I. Antoniadis, "A possible new dimension at a few TeV," Phys. Lett. B, vol. 246, no. 3, pp. 377-384, 1990.

[33] U. Amaldi, W. de Boer, and H. Furstenau, "Comparison of grand unified theories with electroweak and strong coupling-constants measured at LEP," Phys. Lett. B, vol. 260, no. 3-4, pp. 447-455, 1991.

[34] J. Ellis, G. Ridolfi, and F. Zwirner, "Radiative corrections to the masses of supersymmetric Higgs bosons," Phys. Lett. B, vol. 257, no. 1-2, pp. 83-91, 1991.

[35] L. Gammaitoni et al., "Stochastic resonance," Rev. Mod. Phys., vol. 70, no. 1, pp. 223-287, 1998.

[36] G. Marchesini et al. "HERWIG 5.1-A Monte-Carlo event generator for simulating hadron emission reactions with interfering gluons," Comp. Phys. Comm., vol. 67, no. 3, pp. 465-508, 1992.

[37] F. Abe et al., "Observation of top-quark production in (p)over-bar-p collisions with the Collider Detector at Fermilab," Phys. Rev. Lett., vol. 74, no. 14, pp. 2626-2631, 1995.

[38] The Thomson Corporation, ISI Web of Knowledge [Online]. Available: http://scientific.thomson.com/products/jer

[39] F. Sauli, "GEM: A new concept for electron amplification in gas detectors," Nucl. Instrum. Methods Phys. Res. A, vol. A386, no. 2-3, pp. 531-534, 1997.

[40] D. W. O. Rogers et al., "BEAM-A Monte-Carlo code To simulate radiotherapy treatment units," Med. Phys., vol. 22, no. 5, pp. 503-524, 1995.

[41] D. C. Radford, "ESCL8R and LEVIT8R-Software for interactive graphical analysis of HPGe coincidence data sets," Nucl. Instrum. Methods Phys. Res. A, vol. A361, no. 1-2, pp. 297-305, 1995.

[42] Y. Kubota et al., "The CLEO-II detector," Nucl. Instrum. Methods Phys. Res. A, vol. A320, no. 1-2, pp. 66-113, 1992.

[43] A. Ahlbom et al., "Guidelines for limiting exposure to time-varying electric, magnetic, and electromagnetic fields (up to $300 \mathrm{GHz}$ )," Health Phys., vol. 74, no. 4, pp. 494-522, 1998.

[44] A. S. Murray and A. G. Wintle, "Luminescence dating of quartz using an improved single-aliquot regenerative-dose protocol," Radiat. Meas., vol. 32 , no. 1 , pp. $57-73,2000$. 